\title{
Cobordism of exact links
}

\author{
VINCENT BLANLEIL \\ OSAMU SAEKI
}

\begin{abstract}
A $(2 n-1)$-dimensional $(n-2)$-connected closed oriented manifold smoothly embedded in the sphere $S^{2 n+1}$ is called a $(2 n-1)-$ link. We introduce the notion of exact links, which admit Seifert surfaces with good homological conditions. We prove that for $n \geq 3$, two exact $(2 n-1)$-links are cobordant if they have such Seifert surfaces with algebraically cobordant Seifert forms. In particular, two fibered $(2 n-1)-$ links are cobordant if and only if their Seifert forms with respect to their fibers are algebraically cobordant. With this broad class of exact links, we thus clarify the results of Blanlœil [1] concerning cobordisms of odd dimensional nonspherical links.
\end{abstract}

57Q45; 57Q60, 57R65, 57R40

\section{Introduction}

In [1], Blanlœil obtained a lot of important results concerning cobordisms of odd dimensional nonspherical links. Unfortunately, some statements must be clarified, since there are even counterexamples. In fact, when studying knot cobordisms it is very difficult to begin with an arbitrary Seifert surface of a given link. For example, if we consider $S^{n-1} \times D^{n+1}$ embedded trivially in $S^{2 n+1}$ as a Seifert surface of its boundary link $K \cong S^{n-1} \times S^{n}$, then the associated Seifert form is represented by the $0 \times 0$ matrix, since $H_{n}\left(S^{n-1} \times D^{n+1} ; \mathbb{Z}\right)$ vanishes. If there existed a simple link $K^{\prime}$, ie a link $K^{\prime}$ admitting an $(n-1)$-connected Seifert surface, which is cobordant to $K$ and with algebraically cobordant Seifert form (for details, see Section 2), then $K^{\prime}$ would necessarily be diffeomorphic to the sphere $S^{2 n-1}$. This gives a simple counterexample to [1, Propositions 2.1 and 2.2].

In this paper, we will instead use a reasonably broad class of Seifert surfaces, called exact Seifert surfaces, and obtain similar results.

Throughout the paper, we work in the smooth category. All homology and cohomology

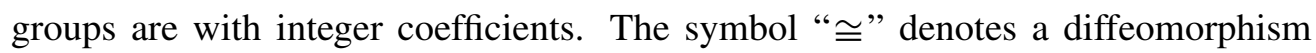
between manifolds or an appropriate isomorphism between algebraic objects. 


\section{Preliminaries}

Let us first recall some definitions.

Definition 2.1 Let $K$ be a closed $(2 n-1)$-dimensional manifold embedded in the $(2 n+1)$-dimensional sphere $S^{2 n+1}$. We suppose that $K$ is $(n-2)$-connected. (We adopt the convention that a space is $(-1)$-connected if it is not empty.) We further assume that it is oriented. Then we call $K$ or its (oriented) isotopy class a $(2 n-1)-$ link, or simply a link.

A link $K$ is a knot if $K$ is a homotopy $(2 n-1)-$ sphere.

Definition 2.2 Two $(2 n-1)$-links $K_{0}$ and $K_{1}$ in $S^{2 n+1}$ are said to be cobordant if there exists a properly embedded oriented $2 n$-dimensional manifold $X$ in $S^{2 n+1} \times[0,1]$ such that

(1) $X$ is diffeomorphic to $K_{0} \times[0,1]$, and

(2) $\partial X=\left(-K_{0} \times\{0\}\right) \cup\left(K_{1} \times\{1\}\right)$,

where $-K_{0}$ is obtained from $K_{0}$ by reversing the orientation.

It is known that for every $(2 n-1)-$ link $K$, there exists a compact oriented $2 n-$ dimensional submanifold $F$ of $S^{2 n+1}$ having $K$ as boundary.

Definition 2.3 Let $K$ be a $(2 n-1)$-link. A compact oriented $2 n$-dimensional submanifold $F$ of $S^{2 n+1}$ having $K$ as its oriented boundary is called a Seifert surface associated with $K$.

Definition 2.4 We say that a $(2 n-1)-$ link is simple if it admits an $(n-1)$-connected Seifert surface.

Definition 2.5 We say that a $(2 n-1)-$ link $K$ is fibered if there exist a smooth fibration

$$
\phi: S^{2 n+1} \backslash K \rightarrow S^{1}
$$

and a trivialization

$$
\tau: N(K) \rightarrow K \times D^{2}
$$

of a closed tubular neighborhood $N(K)$ of $K$ in $S^{2 n+1}$ such that $\left.\phi\right|_{N(K) \backslash K}$ coincides with $\left.\pi \circ \tau\right|_{N(K) \backslash K}$, where $\pi: K \times\left(D^{2} \backslash\{0\}\right) \rightarrow S^{1}$ is the composition of the projection to the second factor and the obvious projection $D^{2} \backslash\{0\} \rightarrow S^{1}$. 
Note that then the closure of each fiber of $\phi$ in $S^{2 n+1}$ is a compact $2 n$-dimensional oriented manifold whose boundary coincides with $K$. We shall often call the closure of each fiber simply a fiber.

Furthermore, we say that a fibered $(2 n-1)-$ link $K$ is simple if each fiber of $\phi$ is $(n-1)$-connected.

Definition 2.6 Suppose that $F$ is a compact oriented $2 n$-dimensional submanifold of $S^{2 n+1}$, and let $G$ be the quotient of $H_{n}(F)$ by its $\mathbb{Z}$-torsion. The Seifert form associated with $F$ is the bilinear form

$$
A: G \times G \rightarrow \mathbb{Z}
$$

defined as follows. For $(x, y) \in G \times G$, we define $A(x, y)$ to be the linking number in $S^{2 n+1}$ of $\xi_{+}$and $\eta$, where $\xi$ and $\eta$ are $n$-cycles in $F$ representing $x$ and $y$ respectively, and $\xi_{+}$is the $n$-cycle $\xi$ pushed off $F$ into the positive normal direction to $F$ in $S^{2 n+1}$.

By definition a Seifert form associated with a $(2 n-1)-$ link $K$ is the Seifert form associated with $F$, where $F$ is a Seifert surface associated with $K$. A matrix representative of a Seifert form with respect to a basis of $G$ is called a Seifert matrix.

Definition 2.7 Let $\mathcal{A}$ be the set of all bilinear forms defined on free $\mathbb{Z}$-modules $G$ of finite rank. Set $\varepsilon=(-1)^{n}$. For $A \in \mathcal{A}$, let us denote by $A^{T}$ the transpose of $A$, by $S$ the $\varepsilon$-symmetric form $A+\varepsilon A^{T}$ associated with $A$, by $S^{*}: G \rightarrow G^{*}$ the adjoint of $S$ with $G^{*}$ being the dual $\operatorname{Hom}_{\mathbb{Z}}(G, \mathbb{Z})$ of $G$, and by $\bar{S}: \bar{G} \times \bar{G} \rightarrow \mathbb{Z}$ the $\varepsilon$-symmetric nondegenerate form induced by $S$ on $\bar{G}=G / \operatorname{Ker} S^{*}$. A submodule $M$ of $G$ is said to be pure if $G / M$ is torsion free, or equivalently if $M$ is a direct summand of $G$. For a submodule $M$ of $G$, let us denote by $M^{\wedge}$ the smallest pure submodule of $G$ that contains $M$. We denote by $\bar{M}$ the image of $M$ in $\bar{G}$ by the natural projection map.

Definition 2.8 Let $A: G \times G \rightarrow \mathbb{Z}$ be a bilinear form in $\mathcal{A}$. The form $A$ is Witt associated to 0 if the rank $m$ of $G$ is even and there exists a pure submodule $M$ of rank $m / 2$ in $G$ such that $A$ vanishes on $M \times M$. Such a submodule $M$ is called a metabolizer for $A$.

Definition 2.9 Let $A_{i}$ : $G_{i} \times G_{i} \rightarrow \mathbb{Z}, i=0,1$, be two bilinear forms in $\mathcal{A}$. Set $G=G_{0} \oplus G_{1}, A=\left(-A_{0}\right) \oplus A_{1}, S=A+\varepsilon A^{T}$, and $S_{i}=A_{i}+\varepsilon A_{i}^{T}, i=0,1$. The form $A_{0}$ is said to be algebraically cobordant to $A_{1}$ if there exist a metabolizer $M$ for $A$ such that $\bar{M}$ is pure in $\bar{G}$, an isomorphism $\varphi: \operatorname{Ker} S_{0}^{*} \rightarrow \operatorname{Ker} S_{1}^{*}$, and an 
isomorphism $\theta$ : Tors $\left(\right.$ Coker $\left.S_{0}^{*}\right) \rightarrow \operatorname{Tors}\left(\right.$ Coker $\left.S_{1}^{*}\right)$ which satisfy the following two conditions:

$$
\begin{aligned}
M \cap \operatorname{Ker} S^{*} & =\left\{(x, \varphi(x)) \mid x \in \operatorname{Ker} S_{0}^{*}\right\} \subset \operatorname{Ker} S_{0}^{*} \oplus \operatorname{Ker} S_{1}^{*}=\operatorname{Ker} S^{*}, \\
d\left(S^{*}(M)^{\wedge}\right) & =\left\{(y, \theta(y)) \mid y \in \operatorname{Tors}\left(\operatorname{Coker} S_{0}^{*}\right)\right\} \\
& \subset \operatorname{Tors}\left(\operatorname{Coker} S_{0}^{*}\right) \oplus \operatorname{Tors}\left(\operatorname{Coker} S_{1}^{*}\right)=\operatorname{Tors}\left(\operatorname{Coker} S^{*}\right),
\end{aligned}
$$

where $d$ is the quotient map $G^{*} \rightarrow$ Coker $S^{*}$ and "Tors" means the torsion subgroup. In the above situation, we also say that $A_{0}$ and $A_{1}$ are algebraically cobordant with respect to $\varphi$ and $\theta$.

Remark 2.10 As pointed out in Blanlœil and Saeki [3] the relation of algebraic cobordism may not be an equivalence relation on the set of all integral bilinear forms of finite rank (see also Vogt [7]). However, it is an equivalence relation on the set of all unimodular bilinear forms of finite rank, as shown in Blanlœil and Michel [2].

\section{Exact links}

Definition 3.1 Suppose $n \geq 2$. A Seifert surface $F$ of a $(2 n-1)-$ link $K$ is said to be exact if the sequence

$0 \rightarrow H_{n}(K) \rightarrow H_{n}(F) /$ Tors $H_{n}(F) \rightarrow H_{n}(F, K) /$ Tors $H_{n}(F, K) \rightarrow H_{n-1}(K) \rightarrow 0$,

derived from the homology exact sequence for the pair $(F, K)$, is well defined and exact. Note that the homomorphism

$$
H_{n}(F, K) / \text { Tors } H_{n}(F, K) \rightarrow H_{n-1}(K)
$$

may not be well defined in general. Here, we impose the condition that this map should be well defined. A $(2 n-1)-$ link is said to be exact if it admits an exact Seifert surface.

Example 3.2 Consider $K=S^{n-1} \times S^{n}$ embedded trivially in $S^{2 n} \subset S^{2 n+1}, n \geq 2$. Then $K$ is a $(2 n-1)-$ link and it bounds two Seifert surfaces $F_{0}=D^{n} \times S^{n}$ and $F_{1}=S^{n-1} \times D^{n+1}$, both of which are embedded in $S^{2 n}$. Then $F_{0}$ is exact, while $F_{1}$ is not, since $H_{n}\left(S^{n-1} \times S^{n}\right) \rightarrow H_{n}\left(S^{n-1} \times D^{n+1}\right)$ is not a monomorphism.

Lemma 3.3 For $n \geq 2$, we have the following.

(1) A simple $(2 n-1)$-link is always exact. In fact, every $(n-1)$-connected Seifert surface is exact.

(2) A fibered $(2 n-1)-$ link is always exact. In fact, every fiber is exact.

(3) A (2n-1)-knot is always exact. In fact, every Seifert surface is exact. 
Proof In the following, let $K$ be a $(2 n-1)-$ link and $F$ a relevant Seifert surface.

(1) Let us consider the exact sequence

$$
H_{n+1}(F, K) \rightarrow H_{n}(K) \rightarrow H_{n}(F) \rightarrow H_{n}(F, K) \rightarrow H_{n-1}(K) \rightarrow H_{n-1}(F) .
$$

Then we have the desired result, since $H_{n+1}(F, K) \cong H^{n-1}(F)=0, H_{n-1}(F)=0$, and $H_{n}(F)$ and $H_{n}(F, K)$ are torsion free.

(2) If $F$ is a fiber of a fibered link, then it is easy to see that $S^{2 n+1} \backslash F$ is homotopy equivalent to $F$. Hence, by Alexander duality, we have

$$
\tilde{H}_{i}(F) \cong \tilde{H}^{2 n-i}(F)
$$

for all $i$, where $\tilde{H}_{*}$ and $\tilde{H}^{*}$ denote reduced homology and cohomology groups, respectively. Consider the exact sequence

$$
\begin{aligned}
0 & \rightarrow \tilde{H}_{n+1}(F) \rightarrow H_{n+1}(F, K) \rightarrow \\
\tilde{H}_{n}(K) & \rightarrow \tilde{H}_{n}(F) \rightarrow H_{n}(F, K) \rightarrow \\
\tilde{H}_{n-1}(K) & \rightarrow \tilde{H}_{n-1}(F) \rightarrow H_{n-1}(F, K) \rightarrow 0 .
\end{aligned}
$$

(Recall that $K$ is $(n-2)-$ connected.) Since

$$
\tilde{H}_{n-1}(F) \cong \tilde{H}^{n+1}(F) \cong H_{n-1}(F, K)
$$

and $\tilde{H}_{n-1}(F) \rightarrow H_{n-1}(F, K)$ is an epimorphism, it must be an isomorphism. Hence $H_{n}(F, K) \rightarrow \widetilde{H}_{n-1}(K)$ is an epimorphism. Furthermore, since

$$
\tilde{H}_{n+1}(F) \cong \tilde{H}^{n-1}(F) \cong H_{n+1}(F, K),
$$

$\tilde{H}_{n+1}(F) \rightarrow H_{n+1}(F, K)$ is a monomorphism, and $\widetilde{H}_{n}(K)$ is torsion free, the homomorphism $\tilde{H}_{n+1}(F) \rightarrow H_{n+1}(F, K)$ must be an isomorphism. Thus $\tilde{H}_{n}(K) \rightarrow \widetilde{H}_{n}(F)$ is a monomorphism. Since $\widetilde{H}_{n}(K)$ is torsion free, the map

$$
\tilde{H}_{n}(K) \rightarrow \tilde{H}_{n}(F) / \text { Tors } \tilde{H}_{n}(F)
$$

is also a monomorphism. Finally, since $\widetilde{H}_{n}(F) \cong \widetilde{H}^{n}(F) \cong H_{n}(F, K)$, we have Tors $\tilde{H}_{n}(F) \cong$ Tors $H_{n}(F, K)$. Then we see easily that the sequence

$0 \rightarrow \tilde{H}_{n}(K) \rightarrow \widetilde{H}_{n}(F) /$ Tors $\widetilde{H}_{n}(F) \rightarrow H_{n}(F, K) /$ Tors $H_{n}(F, K) \rightarrow \widetilde{H}_{n-1}(K) \rightarrow 0$

is well defined and exact.

(3) If $K$ is a homotopy sphere, then $H_{n}(K)=0=\tilde{H}_{n-1}(K)$, and hence

$$
0 \rightarrow \tilde{H}_{n}(F) \rightarrow H_{n}(F, K) \rightarrow 0
$$

is exact. Thus the result is obvious. This completes the proof. 
The following can be regarded as a correction of [1, Proposition 2.1].

Proposition 3.4 Let $K$ be an exact $(2 n-1)-$ link, $n \geq 2$, and $A$ its Seifert form associated with an exact Seifert surface. Then, there exists a simple $(2 n-1)-$ link $K^{\prime}$ cobordant to $K$ such that the Seifert form of $K^{\prime}$ associated with an $(n-1)$-connected Seifert surface is algebraically cobordant to $A$.

Remark 3.5 Note that when $n=1$, every 1 -link admits a connected Seifert surface, and hence is simple.

Proof of Proposition 3.4 Let $F$ be an exact Seifert surface of $K$. By exactly the same method as in Blanlœil[1] and Levine [6], with the help of an engulfing theorem, we can perform embedded surgeries on $F$ inside the disk $D^{2 n+2}$ along spheres $a$ of dimensions $\leq n-1$ embedded in $F$ so that we obtain a simple link $K^{\prime}$ cobordant to $K$ and an $(n-1)$-connected Seifert surface $F^{\prime}$ for $K^{\prime}$.

Let us examine the relationship between the Seifert forms with respect to $F$ and $F^{\prime}$. If the sphere $a$ along which the surgery is performed is of dimension less than or equal to $n-2$, then it does not affect the $n$-th homology of $F$. We again denote by $F$ the result of such surgeries: in particular, $F$ is $(n-2)$-connected. Let us now consider the case where $a$ is of dimension $n-1$. In the following, $[a]$ will denote the homology class in $H_{n-1}(F)$ represented by $a$, where we fix its orientation once and for all.

Case 1 The class $[a]$ has infinite order in $H_{n-1}(F)$.

Since $K$ is exact, the boundary homomorphism $\partial_{*}: H_{n}(F, K) \rightarrow H_{n-1}(K)$ is surjective. By the exact sequence

$$
H_{n}(F, K) \stackrel{\partial_{*}}{\rightarrow} H_{n-1}(K) \stackrel{i_{*}}{\rightarrow} H_{n-1}(F) \stackrel{j_{*}}{\rightarrow} H_{n-1}(F, K),
$$

where $i: K \rightarrow F$ and $j: F \rightarrow(F, K)$ are the inclusions, we see that $j_{*}$ is injective and hence $j_{*}[a]$ has infinite order in $H_{n-1}(F, K) \cong H^{n+1}(F)$. Therefore, there exists an $(n+1)$-cycle $\tilde{a}$ of $F$ such that the intersection number $a \cdot \tilde{a}$ does not vanish. We choose $\tilde{a}$ so that $m=|a \cdot \tilde{a}|(>0)$ is the smallest possible.

Let $\psi: D^{n} \times D^{n+1} \rightarrow D^{2 n+2}$ be the $n$-handle used by the surgery in question such that $\psi\left(S^{n-1} \times\{0\}\right)=a$. As in [1], let us put

$$
F_{T}=F \backslash \operatorname{Int}\left(\psi\left(S^{n-1} \times D^{n+1}\right)\right), \quad F^{\star}=F_{T} \cup \psi\left(D^{n} \times S^{n}\right) .
$$


Let us consider the Mayer-Vietoris exact sequence associated with the decomposition $F=F_{T} \cup \psi\left(S^{n-1} \times D^{n+1}\right)$ :

$$
\begin{aligned}
H_{n+1}(F) \stackrel{s}{\rightarrow} H_{n}\left(\psi\left(S^{n-1} \times S^{n}\right)\right) & \rightarrow H_{n}\left(F_{T}\right) \rightarrow H_{n}(F) \\
& \stackrel{t}{\rightarrow} H_{n-1}\left(\psi\left(S^{n-1} \times S^{n}\right)\right) \stackrel{u}{\rightarrow} H_{n-1}\left(F_{T}\right) \oplus H_{n-1}\left(\psi\left(S^{n-1} \times D^{n+1}\right)\right) .
\end{aligned}
$$

Since the map $s$ is given by the intersection number with $a$, its image coincides with $m \mathbb{Z} \subset \mathbb{Z} \cong H_{n}\left(\psi\left(S^{n-1} \times S^{n}\right)\right)$. Furthermore, since $u$ is an injection, $t$ is the zero map. Therefore, we have the exact sequence

$$
0 \rightarrow \mathbb{Z}_{m} \rightarrow H_{n}\left(F_{T}\right) \rightarrow H_{n}(F) \rightarrow 0 .
$$

Therefore, the inclusion $F_{T} \rightarrow F$ induces an isomorphism

$$
H_{n}\left(F_{T}\right) / \text { Tors } H_{n}\left(F_{T}\right) \rightarrow H_{n}(F) / \text { Tors } H_{n}(F) .
$$

Remark 3.6 In [1], it is stated that the map $s$ is surjective, since the image is generated by the intersection of the $(n+1)-$ cycle dual to $a$ and $\psi\left(S^{n-1} \times S^{n}\right)$. However, such an $(n+1)$-cycle dual to $a$ may not exist, since $\partial F$ may not be a sphere. Here, we used the assumption that $F$ is an exact Seifert surface in order to show that the map $s$ is nontrivial.

Similarly we also have the following exact sequence obtained from the Mayer-Vietoris exact sequence associated with the decomposition $F^{\star}=F_{T} \cup \psi\left(D^{n} \times S^{n}\right)$ :

$$
\begin{aligned}
0 \rightarrow H_{n}\left(\psi\left(S^{n-1} \times S^{n}\right)\right) \rightarrow H_{n}\left(F_{T}\right) \oplus H_{n}\left(\psi\left(D^{n} \times S^{n}\right)\right) \rightarrow H_{n}\left(F^{\star}\right) & \\
& \rightarrow H_{n-1}\left(\psi\left(S^{n-1} \times S^{n}\right)\right) \stackrel{u^{\prime}}{\rightarrow} H_{n-1}\left(F_{T}\right) .
\end{aligned}
$$

Note that the map $u^{\prime}$ is injective, since the image of the composition

$$
H_{n-1}\left(\psi\left(S^{n-1} \times S^{n}\right)\right) \stackrel{u^{\prime}}{\rightarrow} H_{n-1}\left(F_{T}\right) \stackrel{v}{\rightarrow} H_{n-1}(F)
$$

is generated by $[a]$ which is of infinite order, where $v$ is the homomorphism induced by the inclusion. Therefore, we see that the inclusion induces an isomorphism $H_{n}\left(F_{T}\right) \rightarrow H_{n}\left(F^{\star}\right)$.

Summarizing, we have the isomorphisms

$$
H_{n}(F) / \text { Tors } H_{n}(F) \stackrel{\cong}{\longleftarrow} H_{n}\left(F_{T}\right) / \text { Tors } H_{n}\left(F_{T}\right) \stackrel{\cong}{\rightarrow} H_{n}\left(F^{\star}\right) / \text { Tors } H_{n}\left(F^{\star}\right)
$$

induced by the inclusions.

Case 2 The class $[a]$ has finite order in $H_{n-1}(F)$. 
Let us denote the order of $[a]$ by $p>0$. There exists an $n$-chain $\sigma$ in $F$ such that $\partial \sigma=p a$. We may assume that $\sigma$ does not intersect with $a$ outside of its boundary. Then, we have an $n$-chain $\sigma^{\prime}$ in $F_{T}$ such that $\left[\partial \sigma^{\prime}\right]=p\left[\psi\left(S^{n-1} \times\{*\}\right)\right]$ in $H_{n-1}\left(\psi\left(S^{n-1} \times S^{n}\right)\right)$.

As before, we have the exact sequence

$$
H_{n}\left(\psi\left(S^{n-1} \times S^{n}\right)\right) \stackrel{w}{\rightarrow} H_{n}\left(F_{T}\right) \rightarrow H_{n}(F) \rightarrow 0 .
$$

Since $\left[\psi\left(\{*\} \times S^{n}\right)\right] \in H_{n}\left(F_{T}\right)$ has nonzero intersection number with the homology class in $H_{n}\left(F_{T}, \partial F_{T}\right)$ represented by $\sigma^{\prime}$, we see that the map $w$ above is injective. Note that then $(\operatorname{Im} w)^{\wedge}$ is infinite cyclic. Let a generator of $(\operatorname{Im} w)^{\wedge}$ be denoted by $\ell \in H_{n}\left(F_{T}\right)$. Then, we have the exact sequence

$$
0 \rightarrow \mathbb{Z}\langle\ell\rangle \rightarrow H_{n}\left(F_{T}\right) / \text { Tors } H_{n}\left(F_{T}\right) \rightarrow H_{n}(F) / \text { Tors } H_{n}(F) \rightarrow 0,
$$

where $\mathbb{Z}\langle\ell\rangle$ denotes the infinite cyclic group generated by $\ell$. This implies that

$$
H_{n}\left(F_{T}\right) / \text { Tors } H_{n}\left(F_{T}\right) \cong\left(H_{n}(F) / \text { Tors } H_{n}(F)\right) \oplus \mathbb{Z}\langle\ell\rangle .
$$

Similarly, we have the exact sequence

$$
\begin{aligned}
0 \rightarrow H_{n}\left(\psi\left(S^{n-1} \times S^{n}\right)\right) & \rightarrow H_{n}\left(F_{T}\right) \oplus H_{n}\left(\psi\left(D^{n} \times S^{n}\right)\right) \rightarrow H_{n}\left(F^{\star}\right) \\
& \stackrel{t^{\prime}}{\rightarrow} H_{n-1}\left(\psi\left(S^{n-1} \times S^{n}\right)\right) \stackrel{u^{\prime}}{\rightarrow} H_{n-1}\left(F_{T}\right) \rightarrow H_{n-1}\left(F^{\star}\right) \rightarrow 0 .
\end{aligned}
$$

The image of $p$ times the generator of $H_{n-1}\left(\psi\left(S^{n-1} \times S^{n}\right)\right)$ by $u^{\prime}$ vanishes, since it bounds $\sigma^{\prime}$ in $F_{T}$. On the other hand, if $p^{\prime}$ times the generator belongs to $\operatorname{Ker} u^{\prime}$ for some $p^{\prime}$ with $0<p^{\prime}<p$, then the order of $[a]$ is strictly less than $p$, which is a contradiction. Therefore, the image of $s^{\prime}$ is generated by $z=p\left[\psi\left(S^{n-1} \times\{*\}\right)\right]$. Hence, we have the exact sequence

$$
0 \rightarrow H_{n}\left(F_{T}\right) \rightarrow H_{n}\left(F^{\star}\right) \stackrel{t^{\prime}}{\rightarrow} \mathbb{Z}\langle z\rangle \rightarrow 0,
$$

where $\mathbb{Z}\langle z\rangle$ is the infinite cyclic group generated by $z \in H_{n-1}\left(\psi\left(S^{n-1} \times S^{n}\right)\right)$. Let $\eta^{\star}$ be the $n$-cycle in $F^{\star}$ obtained by the union of $p$ times $\psi\left(D^{n} \times\{*\}\right)$ and $\sigma^{\prime}$. Set $\ell^{\star}=\left[\eta^{\star}\right] \in H_{n}\left(F^{\star}\right)$. Then the image of $\ell^{\star}$ by $t^{\prime}$ coincides with $\pm z$. Therefore, we see that $H_{n}\left(F^{\star}\right) /$ Tors $H_{n}\left(F^{\star}\right) \cong\left(H_{n}\left(F_{T}\right) /\right.$ Tors $\left.H_{n}\left(F_{T}\right)\right) \oplus \mathbb{Z}\left\langle\ell^{\star}\right\rangle$.

Summarizing, we have

$$
H_{n}\left(F^{\star}\right) / \text { Tors } H_{n}\left(F^{\star}\right) \cong\left(H_{n}(F) / \text { Tors } H_{n}(F)\right) \oplus \mathbb{Z}\langle\ell\rangle \oplus \mathbb{Z}\left\langle\ell^{\star}\right\rangle .
$$

So, in this case, the rank of the $n$-th homology group increases by two as a result of the surgery. 
In the following, we denote by $F$ the original Seifert surface for $K$ and by $F^{\prime}$ the $(n-1)$-connected Seifert surface for $K^{\prime}$ obtained as a result of the surgeries. Set $G=H_{n}(F) /$ Tors $H_{n}(F)$. Note that

$$
G^{\prime}=H_{n}\left(F^{\prime}\right) / \text { Tors } H_{n}\left(F^{\prime}\right) \cong G \oplus\left(\bigoplus_{i \in \mathcal{I}}\left(\mathbb{Z}\left\langle\ell_{i}\right\rangle \oplus \mathbb{Z}\left\langle\ell_{i}^{\star}\right\rangle\right)\right),
$$

where the indices in $\mathcal{I}$ correspond to the surgeries necessary to kill the torsion of the $(n-1)$-th homology, and $\ell_{i}$ (or $\ell_{i}^{\star}$ ) corresponds to the generator $\ell$ (resp. $\ell^{\star}$ ) above (see Case 2).

Let $A$ (or $A^{\prime}$ ) be the Seifert form for $F$ (resp. $F^{\prime}$ ) defined on $H_{n}(F) /$ Tors $H_{n}(F)$ (resp. $H_{n}\left(F^{\prime}\right) /$ Tors $H_{n}\left(F^{\prime}\right)$ ). Furthermore, let $S$ (or $S^{\prime}$ ) be the intersection form of $F$ (resp. $\left.F^{\prime}\right)$. Note that $\operatorname{Ker} S^{*} \cong H_{n}(K)$ corresponds to $\operatorname{Ker}\left(S^{\prime}\right)^{*} \cong H_{n}\left(K^{\prime}\right)$ under the isomorphism (3-1).

Set $B=(-A) \oplus A^{\prime}$ and $S_{B}=(-S) \oplus S^{\prime}$, which are bilinear forms defined on $G \oplus G^{\prime}$. Note that $G$ can be identified with a submodule of $G^{\prime}$ under the isomorphism (3-1). Let $M$ be the submodule of $G \oplus G^{\prime}$ generated by the elements of the form $(x, x)$ with $x \in G$ and by $\ell_{i}, i \in \mathcal{I}$.

As in [1], we see easily that $M$ is a metabolizer for $B$. Furthermore, $\bar{M}$ is pure in $\overline{G \oplus G^{\prime}}$ and we can easily check that

$$
M \cap \operatorname{Ker} S_{B}^{*}=\left\{(x, x) \in G \oplus G^{\prime} \mid x \in \operatorname{Ker} S^{*}\right\} .
$$

Let $y$ be an arbitrary nonzero element of Tors (Coker $S^{*}$ ). We denote the order of $y$ by $q$. Let

$$
\partial_{*}^{\prime}: G^{*}=H_{n}(F, K) / \text { Tors } H_{n}(F, K) \rightarrow H_{n-1}(K)
$$

be the homomorphism induced by the boundary homomorphism, which is well defined and surjective, since $F$ is an exact Seifert surface. Furthermore, the map

$$
H_{n}(F) / \text { Tors } H_{n}(F) \rightarrow H_{n}(F, K) / \text { Tors } H_{n}(F, K)
$$

induced by the inclusion is identified with $S^{*}$ by virtue of the Poincaré duality, and its image coincides with $\operatorname{Ker} \partial_{*}^{\prime}$. (We also have similar statements for $\left(S^{\prime}\right)^{*}$ as well.) Thus, there exists a $\tilde{y} \in G^{*}$ such that $\partial_{*}^{\prime} \tilde{y}=y$ under the identification Coker $S^{*}=$ $H_{n-1}(K)$. Then, $q(\tilde{y}, \tilde{y}) \in G^{*} \oplus\left(G^{\prime}\right)^{*}$ lies in $S_{B}^{*}(M)$, which implies that $(\tilde{y}, \tilde{y}) \in$ $G^{*} \oplus\left(G^{\prime}\right)^{*}$ lies in $S_{B}^{*}(M)^{\wedge}$. Therefore, we have

$$
d\left(S_{B}^{*}(M)^{\wedge}\right) \supset\left\{(y, y) \mid y \in \text { Tors }\left(\text { Coker } S^{*}\right)\right\}
$$

under the natural identification

$$
\text { Coker } S^{*}=H_{n-1}(K)=H_{n-1}\left(K^{\prime}\right)=\operatorname{Coker}\left(S^{\prime}\right)^{*} .
$$


Lemma 3.7 The order of $d\left(S_{B}^{*}(M)^{\wedge}\right)$ coincides with that of Tors $\left(\operatorname{Coker} S^{*}\right)$.

Proof Since $S_{B}^{*}(M)$ is of finite index in $S_{B}^{*}(M)^{\wedge}$, we can write

$$
S_{B}^{*}(M)^{\wedge} / S_{B}^{*}(M) \cong \bigoplus_{i=1}^{k} \mathbb{Z}_{a_{i}}
$$

where $a_{i}$ are positive integers such that $a_{i}$ divides $a_{i+1}$ for all $i=1,2, \ldots, k-1$, and $k=\operatorname{rank} S_{B}^{*}(M)^{\wedge}$. (Here, we do not exclude the case where $a_{1}=\cdots=a_{r}=1$ for some $r$ with $1 \leq r \leq k$.)

Since $\bar{M}$ is pure in $\overline{G \oplus G^{\prime}}$, we have $S_{B}^{*}\left(G \oplus G^{\prime}\right) \cap S_{B}^{*}(M)^{\wedge}=S_{B}^{*}(M)$ by [2, Section 2]. Therefore, the quotient map $d: G^{*} \oplus\left(G^{\prime}\right)^{*} \rightarrow \operatorname{Coker} S_{B}^{*}$ restricted to $S_{B}^{*}(M)^{\wedge}$ can be identified with the quotient map $S_{B}^{*}(M)^{\wedge} \rightarrow S_{B}^{*}(M)^{\wedge} / S_{B}^{*}(M)$.

Consider $\overline{S_{B}}: \overline{G \oplus G^{\prime}} \times \overline{G \oplus G^{\prime}} \rightarrow \mathbb{Z}$, the $\varepsilon$-symmetric nondegenerate bilinear form induced from $S_{B}$ on $\overline{G \oplus G^{\prime}}=\left(G \oplus G^{\prime}\right) / \operatorname{Ker} S_{B}^{*}$. Since $\bar{M}$ is pure in $\overline{G \oplus G^{\prime}}$, we have a submodule $N$ of $\overline{G \oplus G^{\prime}}$ such that $\overline{G \oplus G^{\prime}}=\bar{M} \oplus N$. Note that $S_{B}^{*}(M)^{\wedge} / S_{B}^{*}(M)$ is naturally isomorphic to $\overline{S_{B}}{ }^{*}(\bar{M})^{\wedge} / \overline{S_{B}} *(\bar{M})$. Therefore, by taking appropriate bases of $\bar{M}$ and $N$, we may assume that a matrix representative of $\overline{S_{B}}$ is of the form

$$
\left(\begin{array}{cc}
0 & D \\
\varepsilon D^{T} & *
\end{array}\right)
$$

where $D$ is the $k \times k$ diagonal matrix with diagonal entries $a_{1}, a_{2}, \ldots, a_{k}$. In particular, the order of

$$
\text { Tors }\left(\operatorname{Coker} S_{B}^{*}\right)=\operatorname{Coker}{\overline{S_{B}}}^{*}={\overline{G \oplus G^{\prime}}}^{*} /{\overline{S_{B}}}^{*}\left(\overline{\left(G \oplus G^{\prime}\right.}\right)
$$

is equal to $\left(a_{1} a_{2} \cdots a_{k}\right)^{2}$.

Note that

$$
{\overline{S_{B}}}^{*}(\bar{M})^{\wedge} /{\overline{S_{B}}}^{*}(\bar{M}) \cong S_{B}^{*}(M)^{\wedge} / S_{B}^{*}(M) \cong \bigoplus_{i=1}^{k} \mathbb{Z}_{a_{i}} .
$$

Therefore, the order of

$$
\text { Coker }{\overline{S_{B}}}^{*}=\operatorname{Coker} \bar{S}^{*} \oplus \operatorname{Coker}{\overline{S^{\prime}}}^{*}
$$

coincides with the square of the order of $\overline{S_{B}} *(\bar{M})^{\wedge} /{\overline{S_{B}}}^{*}(\bar{M})$. Therefore, we have the lemma.

Combining the above lemma with (3-2), we have

$$
d\left(S_{B}^{*}(M)^{\wedge}\right)=\left\{(y, y) \mid y \in \text { Tors }\left(\operatorname{Coker} S^{*}\right)\right\} .
$$


Therefore, we conclude that $A$ and $A^{\prime}$ are algebraically cobordant. This completes the proof of Proposition 3.4.

As a corollary, we have the following, which can be regarded as a correction of [1, Proposition 2.2].

Proposition 3.8 Let $K$ be an exact $(2 n-1)-l i n k, n \geq 3$, and $A$ its Seifert form associated with an exact Seifert surface. Then, there exists a simple $(2 n-1)-$ link $K^{\prime}$ cobordant to $K$ such that the Seifert form of $K^{\prime}$ associated with an $(n-1)$-connected Seifert surface coincides with $A$.

Proof By Proposition 3.4, there exists a simple $(2 n-1)$-link $K^{\prime \prime}$ cobordant to $K$ such that the Seifert form $A^{\prime \prime}$ of $K^{\prime \prime}$ associated with an $(n-1)$-connected Seifert surface is algebraically cobordant to $A$. On the other hand, it is known that there exists a simple $(2 n-1)$-link $K^{\prime}$ whose Seifert form associated with an $(n-1)$-connected Seifert surface coincides with $A$. Since $A$ and $A^{\prime \prime}$ are algebraically cobordant, we see that $K^{\prime}$ and $K^{\prime \prime}$ are cobordant by [2]. Then, $K$ and $K^{\prime}$ are cobordant, and the desired result follows.

Remark 3.9 We do not know if the above proposition holds also for $n=2$ or not.

For exact links, we have the following, which can be regarded as a correction of [1, Théorème 1].

Theorem 3.10 Let $K$ and $K^{\prime}$ be exact $(2 n-1)-$ links, $n \geq 3$. If their Seifert forms with respect to exact Seifert surfaces are algebraically cobordant, then $K$ and $K^{\prime}$ are cobordant.

Proof By Proposition 3.8, there exists a simple $(2 n-1)$-link $\tilde{K}$ (or $\tilde{K}^{\prime}$ ) cobordant to $K$ (resp. $K^{\prime}$ ) such that the Seifert form of $\widetilde{K}$ (resp. $\left.\widetilde{K}^{\prime}\right)$ with respect to an $(n-1)-$ connected Seifert surface coincides with the Seifert form of $K$ (resp. $K^{\prime}$ ) with respect to an exact Seifert surface. By our assumption, the Seifert forms of $\widetilde{K}$ and $\widetilde{K}^{\prime}$ are algebraically cobordant. Then, by [2], we see that $\tilde{K}$ and $\tilde{K}^{\prime}$ are cobordant. Therefore, $K$ and $K^{\prime}$ are cobordant. 


\section{Cobordism of fibered links}

The following can be regarded as a correction of [1, Théorèmes 2 et $\mathrm{A}]$.

Theorem 4.1 Let $K$ and $K^{\prime}$ be two fibered (2n-1)-links, $n \geq 3$. Then, $K$ and $K^{\prime}$ are cobordant if and only if their Seifert forms with respect to their fibers are algebraically cobordant.

Proof By Lemma 3.3, a fiber of a fibered link is always exact. Thus, by Theorem 3.10, if the Seifert forms with respect to the fibers are algebraically cobordant, then $K$ and $K^{\prime}$ are cobordant.

Conversely, suppose that $K$ and $K^{\prime}$ are cobordant. Let $A$ (or $A^{\prime}$ ) be the Seifert form of $K$ (resp. $K^{\prime}$ ) with respect to a fiber. By Proposition 3.8 and Lemma 3.3, there exists a simple $(2 n-1)-$ link $\widetilde{K}$ (or $\tilde{K}^{\prime}$ ) cobordant to $K$ (resp. $K^{\prime}$ ) such that the Seifert form with respect to an $(n-1)$-connected Seifert surface coincides with $A$ (resp. $A^{\prime}$ ). Since $A$ and $A^{\prime}$ are unimodular, we see that $\widetilde{K}$ and $\widetilde{K}^{\prime}$ are fibered (see Durfree [4] and Kato [5]). Since $K$ and $K^{\prime}$ are cobordant, we see that $\widetilde{K}$ and $\tilde{K}^{\prime}$ are also cobordant. Then, by Blanlœil and Michel [2], we see that $A$ and $A^{\prime}$ are algebraically cobordant. This completes the proof.

We finish with an open problem.

Question 4.2 Does there exist a link which is not exact?

Acknowledgements The authors would like to thank the anonymous referee for helpful comments. The second author would like to express his thanks to the people at IRMA, University of Strasbourg, France, for their hospitality during the preparation of the manuscript. The second author has been partially supported by the University of Strasbourg, France, by FY 2008 Researcher Exchange Program between JSPS and CNRS, and by Grant-in-Aid for Scientific Research (No. 23244008), JSPS.

\section{References}

[1] V Blanlœil, Cobordisme des entrelacs, Ann. Fac. Sci. Toulouse Math. 7 (1998) 185-205 MR1656167

[2] V Blanlœil, F Michel, A theory of cobordism for non-spherical links, Comment. Math. Helv. 72 (1997) 30-51 MR1456314

[3] V Blanlœil, O Saeki, Cobordism of fibered knots and related topics, from: "Singularities in geometry and topology 2004", (J-P Brasselet, T Suwa, editors), Adv. Stud. Pure Math. 46, Math. Soc. Japan, Tokyo (2007) 1-47 MR2342884 
[4] A H Durfee, Fibered knots and algebraic singularities, Topology 13 (1974) 47-59 MR0336750

[5] M Kato, A classification of simple spinnable structures on a 1-connected Alexander manifold, J. Math. Soc. Japan 26 (1974) 454-463 MR0362328

[6] J Levine, Knot cobordism groups in codimension two, Comment. Math. Helv. 44 (1969) 229-244 MR0246314

[7] R Vogt, Cobordismus von hochzusammenhängenden Knoten, Bonn Math. Publ. 116, Univ. Bonn Math. Institut (1980) MR578661

IRMA Université de Strasbourg

7, rue René Descartes, 67084 Strasbourg cedex, France

Institute of Mathematics for Industry, Kyushu University

Motoka 744, Fukuoka 819-0395, Japan

v.blanloeil@math.unistra.fr, saeki@imi.kyushu-u.ac.jp

http://www-irma.u-strasbg.fr/ blanloei/,

http://imi.kyushu-u.ac.jp/ saeki/

Received: 17 November $2011 \quad$ Revised: 16 March 2012 